\title{
Gender Classification Based on Iris Recognition Using Artificial Neural Networks
}

\author{
$1^{\text {st }}$ Basna Mohammed Salih Hassan \\ Technical College of Informatics Akre, \\ Duhok Polytechnic University \\ Duhok, Iraq \\ basna.mhmed@dpu.edu.krd
}

\author{
$2^{\text {nd }}$ Adnan Mohsin Abdulazeez \\ Research Center \\ Duhok Polytechnic University \\ Duhok, Iraq \\ adnan.mohsin@dpu.edu.krd
}

\author{
$3^{\text {rd }}$ Omer Mohammed Salih Hassan \\ Research Center \\ Duhok Polytechnic University \\ Duhok, Iraq \\ omar.mhamed@dpu.edu.krd
}

https://doi.org/10.48161/qaj.v1n2a63

\begin{abstract}
Biometric authentication is one of the most quickly increasing innovations in today's world; this promising technology has seen widespread use in a variety of fields, including surveillance services, safe financial transfers, creditcard authentication. in biometric verification processes such as gender, age, ethnicity is iris recognition technology is considered the most accurate compared to other vital features such as face, hand geometry, and fingerprints. Because the irises in the same person are not similar. In this work, the study of gender classification using Artificial Neural Networks (ANN) based on iris recognition. The eye image data were collected from the IIT Delhi IRIS Database. All datasets of images were processed using various image processing techniques using the neural network. The results obtained showed high performance in training and got good results in testing. ANN's training and testing process gave a maximum performance at $96.4 \%$ and $97 \%$ respectively.
\end{abstract}

Keywords - Gender prediction, Iris biometrics, Artificial Neural Networks, Canny Edge Detection.

\section{INTRODUCTION}

For gender Prediction, many unique biological features can be used, such as facial features, fingerprints, voice, retina, and iris, but the iris image features for gender prediction are more robust than other frameworks in the biometric authentication system[1], [2] [3]. Many countries use the iris recognition system for passports, forensic medicine, etc [4], [5][6].

The iris recognition system for identification is one of the most used techniques to maintain privacy and security in the field of protection systems such as fingerprint, voice or signature technologies, and other biometric technologies that are present in the person and his entity and cannot be changed[7], [8]. Iris recognition systems are at the top of the pyramid for these technologies, due to many of the features that make them go a long way in front of the rest of these technologies in terms of accuracy and safety, for example, we have not found, to this day, two people with the same iris as it does not change with the time or affected by circumstances External as in handprints, so it is considered one of the best biometric methods used in this field and now this technology has begun to spread and to increase its use in many areas of daily life, as in airports, banks, and others. The iris recognition system is done in several steps after obtaining an image of the eye, segmentation of the iris to extract features, and then matching patterns. (shown fig 1) [9][10], [11].

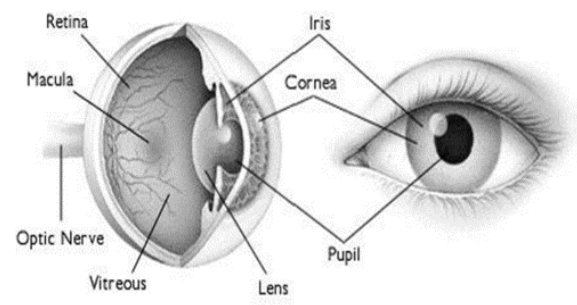

Fig1: the human eye[12]

of brain neurons to process data and accomplish tasks in various fields, and they are the most popular patterns and methods of machine learning aimed at providing algorithms and software capable of learning by experience[13], [14]. When it is said that neural networks mimic the mechanism of action of vital neurons in the brain, this means two things: The first is a structural one concerned with forming the neural network to consist of a certain number of nodes, each of which is called a "neuron" linked with each other through artificial connections. The second thing is the behavioral aspect. That is, artificial neurons mimic vital neurons in how they generate and transmit signals between them, and the neuronal network as a whole must mimic the work of the brain in terms of its ability to learn from experience, and the more experience gained, the greater the strength of the synapses between vital neurons, and thus our performance in carrying out a task What better. The same should be true of artificial neural networks [15][16], [17].

In general terms, a neural network takes the form of multiple layers, each of which has a certain number of neurons. The least possible number of layers is three: the input layer, the output layer, and the hidden layer (or layers). The input layer receives the data that must be processed, and according to the input data values, certain neurons will be activated within the input layer, which in turn will activate the hidden layer neurons, and so on up to the output layer through which we obtain the decision related to the task to be performed. A neural network consists of neurons arranged in layers, with each neuron in each layer communicating with the neurons of 
the previous and next layer [18], [19] [20]-[22]. (shown fig. 2)

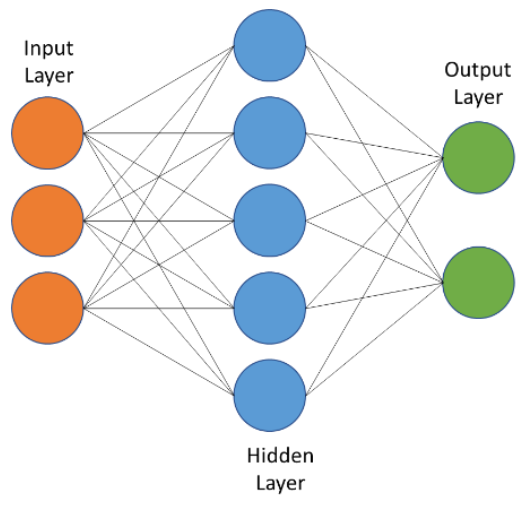

Fig.2 Artificial Neural Network $(A N N)[19]$

Canny Edge detection was first suggested by JOHN CANNY in 1986 [23]. Edge detection is a technique that extracts useful structural information from various visual objects and greatly reduces the amount of data to be processed. And it has been widely used in various computer vision systems. Kanye found that the edge detection requirements are similar in different vision systems, therefore, the wide application significance of edge detection technology can be realized. General standards for edge detection include the following:

$\checkmark$ The maximum number of edges in the captured image should be captured as accurately as possible to reduce the error rate.

$\checkmark \quad$ The exposed edge should be placed precisely in the center of the rear edge.

$\checkmark$ Defined edges in the image must be marked only once, and wherever possible, image noise should not produce false edges.

To meet these requirements, Canny used a varied method. The optimum function in Canny's detector is described by the sum of four exponential terms, which can be approximated by the first derivative of the Gaussian function. Among the commonly used edge detection methods at present, the edge detection algorithm is precisely selected and it can provide a good and reliable detection method. Since it has the advantages of meeting the three criteria for edge detection and a simple implementation process, it has become one of the most popular algorithms for edge detection. The edge detection algorithm can be divided into 5 steps:

1. Use the Gaussian filter to soften the image and filter out the noise.

2. Calculate the gradient intensity and orientation of each pixel in the image.

3. A non-maximum suppression is applied to eliminate the spurious response caused by edge detection.

4. Double threshold detection is used to determine true and potential edges.

5. Edge detection is finally completed by blocking weak isolated edges.

In this work, an iris recognition study for biometric identification and safety applications using ANN is presented. The application of backpropagation artificial neural networks (ANN) is discussed and implemented in this work. The iris images were obtained from bank online datasets to suit the proposed target.

This paper discussed gender prediction from iris image data in the IIT Delhi IRIS Database using artificial neural networks (ANN). In section, I introduction of the basic concepts, section II reviewing the latest literature during the past four years, section III the steps of the approach taken, section IV presenting the results of the approach taken, and a table reviews the most recent papers and results obtained from the techniques used and their comparison in a table and discussing each technique and its challenges, Section V Conclusions drawn from the research.

\section{LITERATURE REVIEW}

Gender prediction strategies based on iris detection are commonly employed in the process of human identity, specifically biometric identification. Below is a review of the latest paper on gender recognition:

A. Kuehlkamp (2018) [24], focused on determining if the details for prediction of gender are included in the iris stroma texture, the eye contour region texture, or both, using linear SVM and CNN. The results indicate that detail specifying the gender is mostly in the periocular region.

K. Ito \& et al. (2019) [25], proposed a prediction of age and gender from face images using convolutional neural networks (CNN). WideResNet has the highest record of age and gender forecasting relative to other architectures, where age by regression is estimated and gender by classification is estimated.

Sreya K C (2020) [26], Presented a process for the prediction of gender using (ANN). Through a certain training process and more processing to obtain better accuracy. to identified gender classification extracted features the area, center, and bounding box from iris images. In the area and bounding box of normalized iris images, the male values are the largest.

B. Fang et al. [27], pointed that the similarities between gender irises are indistinguishable using classic Dogman approaches and can be discovered through deep learning. Used a convolutional neural network (CNN) with two separate datasets of the iris to find the relationship between the left and right irises of the same person or different persons.

S. Arora and M. P. S. Bhatia (2018) [28], aimed to determine the gender of faces using an approach based on convolutional neural networks (CNNs) on the publicly available CASIA dataset for face recognition. The results on CASIA benchmark demonstrate that superior classification performance can be achieved by using (CNNs) for gender recognition.

A. Rattani \& et al. (2018) [29], studied the prediction of the genus from RGB optic images captured by mobile using deep learning. Pre-trained convolutional neural network (CNN) architectures dedicated to gender prediction were implemented. To improve the accuracy of prediction, multiclassifier fusion was used.

A. Grazia Mignani \& et al. (2016)[30], overcame causing misclassification when applying a Binary Statistical Features (BSIF) algorithm because it can create synthetic tissue, a modified BSIF method of genus classification from iris tissue images captured with NIR sensors was implemented. The latter had better gender classification results $(94.6 \%, 91.33 \%$ ) left and right eye respectively. 


\section{Methodology}

the process of gender prediction can be made from the identification of the iris of the eye[31], Through the following stages (as shown in fig.3):

- Eye image

- Split digital image

- $\quad$ Change the range of pixel density values (contrast stretching or normalization technique)

- Extract attribute, Artificial Neural Networks (ANN)

- $\quad$ Ranking

- Train model

- Recognition decision

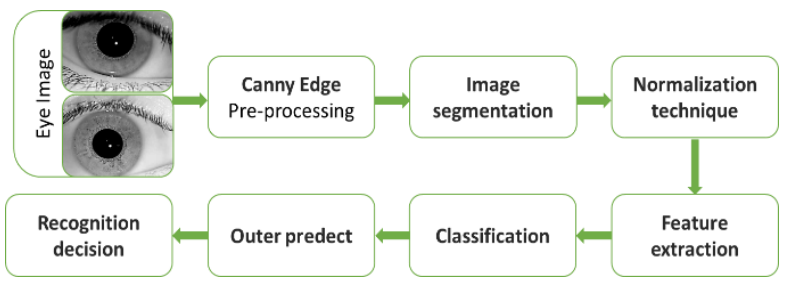

Fig.3 block diagram of gender prediction [32]

\section{A. Prepare the data}

Acquire digital images and perform processing operations such as filtering, segmentation, and image normalization to extract important features from the iris with high accuracy using a modified Canny edge detection algorithm [33]. Shown fig. 4.

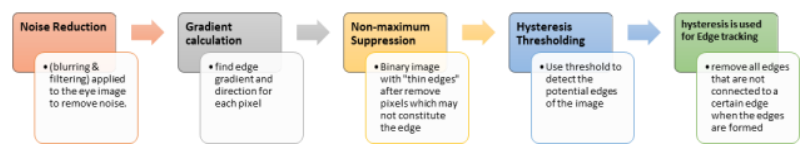

Fig.4 Step of the canny algorithm[34]

The image is processed by applying (blurring \& filtering) to remove noise, improve the appearance of the image, detect the gradation of the edge and the direction of each pixel, to convert it into a binary image with thin edges after removing pixels that may not form the edge. Use threshold to discover possible edges of an image and then remove discontinuous edges [34], [35]. Shown fig.5.

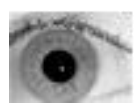

a. Original Image

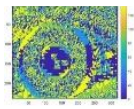

b.

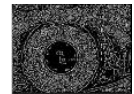

c. magnitude

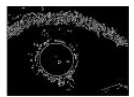

d.Final

Edge image
Fig. 5 edge detection

\section{B. Segmentation}

Segmentation of the iris is the main step in completely identifying the iris. Its objective is to distinguish the functional iris pattern from other parts of the eye and noise [36]. The precision with which the iris is segmented will have a significant impact on the results of future treatment [37].

\section{Normalization technique}

After segmenting the iris, the image must be normalized and brought into a similar range of gradient. Since the optical scale of the iris, the pupil location inside the iris, and the direction of the iris vary between individuals, it is necessary to normalize the iris picture such that it is universally represented with equivalent measurements. The normalization method was used to unwrap the iris and turn it into its polar counterpart. When considering that the center of the pupil in the eye picture was the reference point [38], [39].

\section{Extract attribute (ANN)}

Neural networks are complex neuron architectures that can accept several inputs to generate one output. This is a neural network's primary function - to transform inputs into useful outputs. Normally a neural network consists of one or more hidden layers in the input and output system [40]. all neurons influence each other in a neural network and are therefore interrelated. The network will recognize and track any part of the information set in hand and the relationship between the various data items. This is how neural networks in huge amounts of data will find very complicated patterns. Each node has a weight and is bound by another node on the next layer. Based on their relative value compared with other inputs, neurons are allocated weight. Data may be fed into the networks from input graphical analysis and trained to produce particular outputs [41].

\section{E. Classification}

The project is implemented through artificial neural networks, the backpropagation algorithm is a commonly employed method in which the optimum performance is calculated for each input. Very useful for advanced feed networks. Backpropagation involves the process of stimulation of the artificial neurons (or "nodes"). There are two components: preparation and research. The initial layer is the network entry layer, and the final output layer is the network output layer [42]. The rest of the layers is called the hidden layers. The layers are connected with weights by knots. Training is carried out through a series of training data each with a related target output for controlled formation on a neural network. Finally, the grayscal image of the normal eye becomes binary and the characteristics are removed throughout the testing phase. Take the qualified data to determine if the test iris was male or female [43], [44].

Two units are made up of each neuron. The first unit adds the input signal products and the weight coefficients. The other unit is a nonlinear process called the activation feature of the neuron. To teach the neural network, a training data set is needed. Inputs consistent with goal success are used in the training data collection [45]. The first stage is to start the method of teaching with a high parameter value. In the second step, the weight coefficients are defined and the parameter is increasingly reduced [19], [46].

\section{DATA SET}

The IIT Delhi IRIS database comprises iris photographs taken from IIT Delhi, New Delhi, India, students, and employees. In January-July 2007, this database was acquired by JIRIS, JPC1000, optical CMOS camera in Biometrics Research Laboratory. The accessible archive is made by 224 users and 
all images are bitmapped (*.bmp). The demographic range of all topics in the database comprises 176 men and 48 women between the ages of 14 and 55 years. The 1120 image database is divided into 224 separate directories with an integer identification/number each. These images are $320 \times 240$ pixels in size and all these pictures have been taken indoors [47].

\section{EXPERIMENTAL RESULTS AND DISCUSSION}

The IIT Delhi IRIS image dataset. A dataset of iris images classified by gender and age, 48 images for women and 176 images for men. To ensure diversity of experimental data, the mixed iris samples were universal and sufficient. The IIT Delhi dataset contains 2240 images representing 224 persons. The resolution of these images is 320 x 240 pixels. In this work, selected Randomly extracted 60 samples as experimental samples. 30 samples for females and 30 samples for males.

To detect the edges of the iris, the Canny edge detection algorithm is used, then by Gaussian blurring to remove noise, and finally, the Circular Hough transform is applied to the Gaussian blurred image to detect the limbic boundary area, interpolation method applied in MATLAB functions [48]. The effect of Canny edge detection is shown in fig. 7, and the Gaussian blurred picture is shown in fig 8 . The limbic border is plotted on the iris picture using the center and radius values returned as in fig 9. Iris image is cropped to fit the limbic boundary in that image, as shown in fig. 10. The cropped image is binarized using thresholds to achieve the pupil region. To obtain the pupillary region depicted in Fig.11, morphological closing, eroding, and opening are used. Thus, normalized images of 50 eyes were created to construct a training database [49], [50].

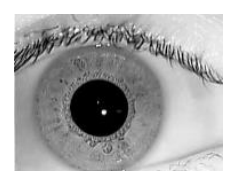

Fig. 6 original image

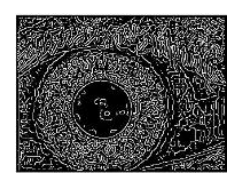

Fig. 7 edge detected

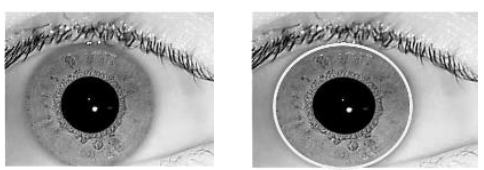

Fig. 9 basic feature extraction

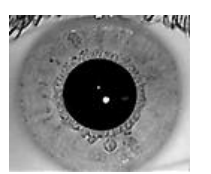

Fig. 10 iris

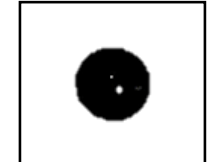

Fig. 11 pupillary region

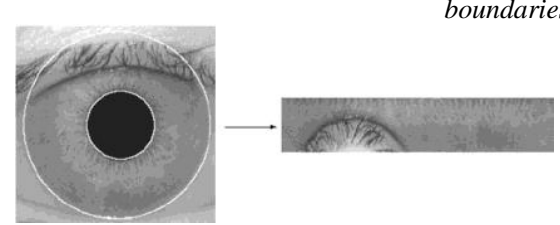
pupillary bundaries

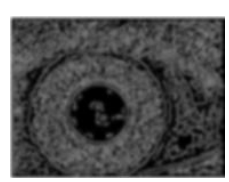

Fig.8 gaussian

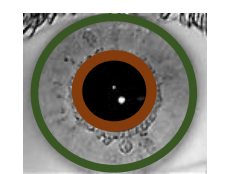

Fig.12 limbic and
Fig.13 normalized iris image
Eight features were extracted from the image set selected for training artificial neural networks (ANN) and the mean, contrast, and standard deviation of the images are calculated to obtain additional features for training the network, the neural network input is the eye feature data set, and the gender data set is the neural network output [51]. After processing the image, all the database images are converted into one-dimensional vectors to feed the neural network. All these vectors are fed one by one to the network in succession during training and testing operations along with the target values for each image[52]. The neural network is supposed to establish the input-output relationships between the images and their targets during the training process. Repeat the training process and adjust the neural weights in the right direction to reduce error and the network is ready for use for testing or recognition. The test is similar to training in all parts except for the absence of the weight adjustment part. The resulting outputs are used as the final evaluations of the network [53].

A basic neural network is built of 30 hidden layers, eight input, and one output. Epoch 500 and 0.01 learning rate. In our system, 10 images have been used for each of 60 Iris people. For training used 6 iris image and used 4 iris images for artificial neural networks (ANN) testing. Shown fig.14.

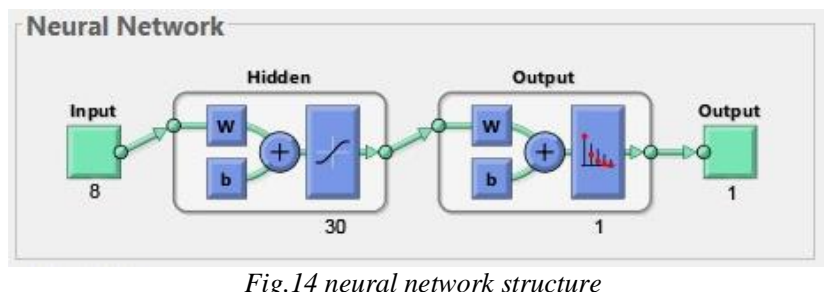

It is a feed-forward NN with 30 neurons, 500 epochs, and a learning rate of 0.01 . A single output is provided by 8 features input to a neural network. the network took 0.7 seconds to reach the MSE of 0.042 . The training took 500 epochs. Hidden layer sizes and types were chosen based on more than 30 different trials with different layer functions and sizes until the best performance criteria were met. The qualified network's efficiency reached $96.4 \%$ in the training, while the test reached $97 \%$. Shown fig. 15 .

The neural network was trained using different parameters. The various criteria altered the preparation and test outcomes. The third experiment achieved the highest training score of $96.4 \%$, while the evaluation performance hit $98 \%$. The parameters of the neural network and their associated training and test outcomes are seen in Table 1.

TABLE I. PERFORMANCE NEURAL NETWORK (NN) WITH DIFFERENT PARAMETERS

\begin{tabular}{|c|c|c|c|c|}
\hline $\begin{array}{c}\text { Learning } \\
\text { rate }\end{array}$ & $\begin{array}{c}\text { Momentum } \\
\text { factor }\end{array}$ & $\begin{array}{c}\text { Bias } \\
\text { values }\end{array}$ & $\begin{array}{c}\text { Training } \\
\text { performance }\end{array}$ & $\begin{array}{c}\text { Test } \\
\text { performance }\end{array}$ \\
\hline 0.01 & 0.002 & $<0.01$ & $94.2 \%$ & $86 \%$ \\
\hline 0.001 & 0.2 & $<0.01$ & $94.8 \%$ & $96 \%$ \\
\hline 0.001 & 0.1 & $<0.10$ & $94 \%$ & $97 \%$ \\
\hline 0.001 & 0.1 & $<0.5$ & $94 \%$ & $67 \%$ \\
\hline 0.001 & 0.2 & $<0.2$ & $95 \%$ & $91 \%$ \\
\hline
\end{tabular}




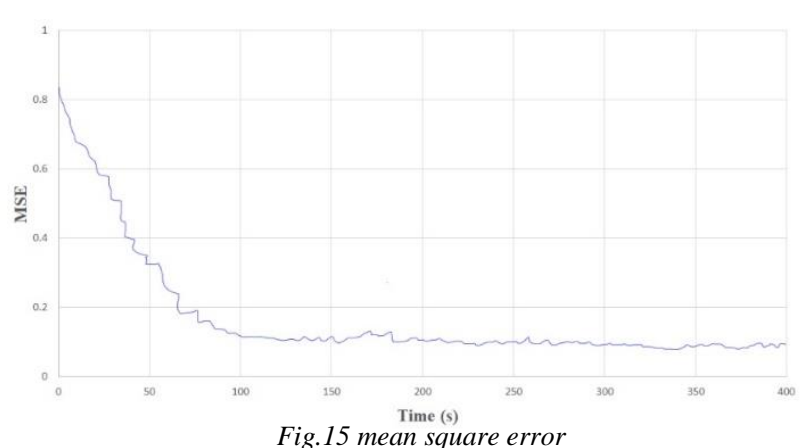

Fig. 15 mean square error

Loss

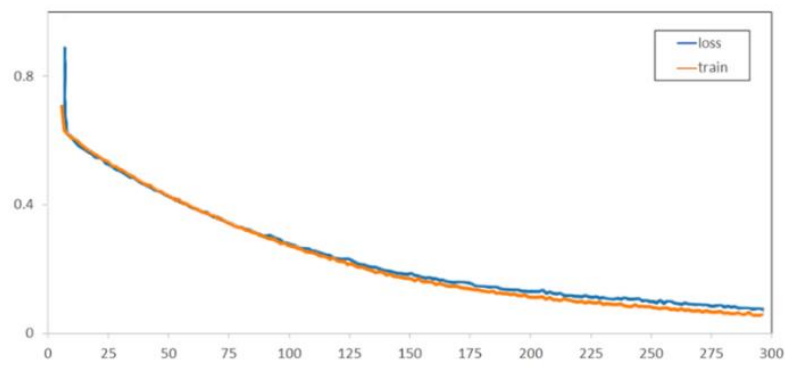

Accuracy

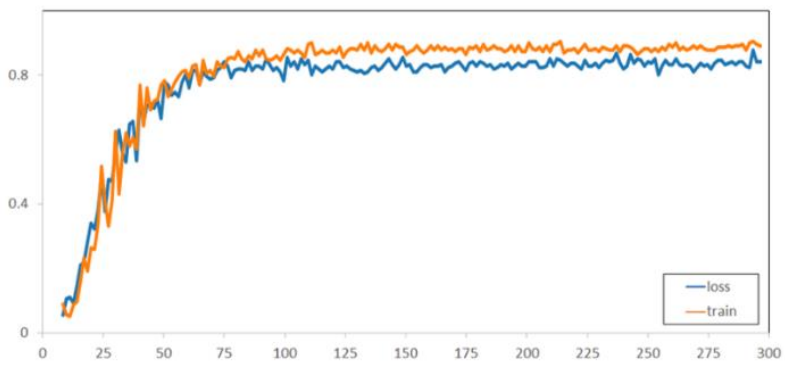

Fig.16 Loss and Classification Accuracy over Training

\section{COMPARATIVE STUDIES}

Table 2 shows a comparison of the gender prediction from the previous research study of some of the current and proposed work performance. A comparison of the different matching and ratings was provided based on overall performance.

TABLE II. COMPARISON OF THE GENDER PREDICTION

\begin{tabular}{|c|c|c|c|c|}
\hline $\operatorname{Ref}$ & $\begin{array}{l}\text { Feature's } \\
\text { extraction } \\
\text { algorithm }\end{array}$ & $\begin{array}{c}\text { Classification } \\
\text { algorithm }\end{array}$ & Data sets & Accuracy \\
\hline $\begin{array}{l}\text { J. Tapia \& et } \\
\text { al. (2019) [54] }\end{array}$ & $\overline{\text { SRCNNs }}$ & random-forest & $\begin{array}{l}\text { captured } \\
\text { from the } \\
\text { iPhone } \\
\text { camera }\end{array}$ & $\begin{array}{l}90.15 \% \text { (right } \\
\text { eye) } \\
87.15 \% \text { (left } \\
\text { eye) }\end{array}$ \\
\hline $\begin{array}{c}\text { C. O. Lawal \& } \\
\text { et al. (2019) } \\
{[55]}\end{array}$ & $\mathrm{CNN}$ & $\begin{array}{c}\text { neural network } \\
\text { CNN }\end{array}$ & $\begin{array}{l}6000 \text { images } \\
\text { of Nigerians }\end{array}$ & $98.72 \%$ \\
\hline $\begin{array}{l}\text { M. Sandhya et } \\
\text { al. (2021) [56] }\end{array}$ & $\mathrm{CNN}$ & $\begin{array}{c}\text { Deep learning } \\
\text { SVM }\end{array}$ & Poly-U & $93.8 \%$ \\
\hline \multirow{4}{*}{ 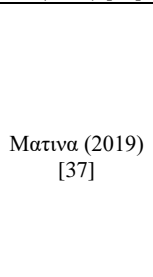 } & \multirow{4}{*}{$\begin{array}{c}\text { CNN (VGG- } \\
16)\end{array}$} & \multirow{4}{*}{$\begin{array}{c}\text { CNN (VGG- } \\
16)\end{array}$} & IITD & $100 \%$ \\
\hline & & & $\begin{array}{l}\text { CASIA-Iris- } \\
\text { V1 }\end{array}$ & $98.3 \%$ \\
\hline & & & $\begin{array}{l}\text { CASIA-Iris- } \\
\text { thousand }\end{array}$ & $95 \%$ \\
\hline & & & $\begin{array}{l}\text { CASIA-Iris- } \\
\text { Interval }\end{array}$ & $91 \%$ \\
\hline $\begin{array}{l}\text { Sreya K C, } \\
(2020)[26]\end{array}$ & $\begin{array}{l}\text { Canny edge } \\
\text { detection }\end{array}$ & ANN - SVM & IITD & $\begin{array}{c}\text { value of the } \\
\text { iris area and } \\
\text { bounding box } \\
\text { of females }\end{array}$ \\
\hline
\end{tabular}

\begin{tabular}{|c|c|c|c|c|}
\hline & & & & $\begin{array}{c}\text { smaller than } \\
\text { males }\end{array}$ \\
\hline $\begin{array}{l}\text { B. Fang et al. } \\
\text { (2019)[27] }\end{array}$ & $\begin{array}{l}\text { Daugman's } \\
\text { rubber }\end{array}$ & $\begin{array}{l}\text { CNN (VGG- } \\
16)\end{array}$ & $\begin{array}{l}\text { CAS-SIAT } \\
\text { CASIA-Iris- } \\
\text { Interval }\end{array}$ & $94 \%$ \\
\hline \multirow{3}{*}{ (2019) [57] } & \multirow{3}{*}{$\mathrm{CNN}$} & $\mathrm{CNN}$ & \multirow{3}{*}{$\begin{array}{l}\text { thirteen } \\
\text { forest- } \\
\text { vegetation } \\
\text { specie }\end{array}$} & $99 \%$ \\
\hline & & ANN & & $94 \%$ \\
\hline & & SVM & & $91 \%$ \\
\hline$(2018)[58]$ & $\begin{array}{l}\text { S1 \& S2 } \\
\text { sound }\end{array}$ & $\begin{array}{l}\text { ANN } \\
\text { CNN }\end{array}$ & $\begin{array}{l}\text { PASCAL } \\
\text { Btraining }\end{array}$ & $97.9 \%$ \\
\hline (2020) [59] & $\begin{array}{l}\text { polyp edges } \\
\text { and non- } \\
\text { polyp edges }\end{array}$ & $\begin{array}{l}\mathrm{CNN}+\mathrm{ANN} \\
\mathrm{CNN}+\mathrm{SVM}\end{array}$ & Kvasir & $97.9 \%$ \\
\hline $\begin{array}{l}\text { Proposed } \\
\text { work }\end{array}$ & $\begin{array}{l}\text { Canny edge } \\
\text { detection }\end{array}$ & ANN & IITD & $96.4 \%$ \\
\hline
\end{tabular}

CASIA data set is one of the most widely used datasets[37], [58], as well as the IITD datasets [26], [37]. That the accuracy of the algorithm's performance depends on several aspects, including the accuracy of the image in the database and the processing methods to extract the features later. Neural network techniques have proven classifiers to train the machine for the most accurate prediction [57].

ANN is a computing system that can be learned to conduct different activities, including the approximation of functions, classification, and data clustering [60]. One of the primary benefits of neural networks over classical statistical approaches used in previous iris classification studies is that they do not need advanced awareness of the data's probability distribution; rather, they learn the statistical distribution of each class through preparation. The neural network generates multidimensional judgment boundaries dependent on the properties of the training data. Additionally, it extracts all required features for the problem statement and feature selection and selects the critical features that increase the deep learning model's efficiency [61], [62].

When solving the issue of image classification (ANN), a 2dimensional image must be transformed into a onedimensional vector before model training. And two drawbacks are created by that: (i) As the picture size grows, the amount of trained parameters is increasing significantly. (ii) ANN loses the image's spatial characteristics. Spatial characteristics apply to pixel organization in an image. In input data needed to manage sequence data, ANN cannot record the sequential details [26].

But CNN captures the image's space characteristics. Spatial characteristics refer to the pixel structure and the image relationship. It helps us to correctly identify the item, its position, and its connection to other artifacts in an image. The principle of parameter sharing is now adopted by CNN [63]. A single filter is applied to produce a function map across various parts of the input. CNN automatically learns the filters without direct mention of them. These filters help to extract the correct and relevant features from the input data. CNN often performs well on sequential inputs [27], [37], [55], [56]. CNNs are used together with ANNs to serve as layers of classification or regression, although this depends on the issue statement [57]-[59].

\section{CONCLUSION}

The detection of Iris in today's world of IT is recognized by the unique features of the human iris as an efficient way of authenticating and identifying biometric ID systems in the business world. Iris recognition is one of the most powerful ways of biometric verification and identification, due to the 
unique features of the human iris. This paper addressed and applied the use of the backpropagation ANN for Gender Recognition Classification. To ensure the accuracy of the neural network in iris recognition all datasets of images were processed using various image processing techniques using the neural network tool in MATLAB. The obtained results have shown high performance and given good results. The training process of the ANN has given a maximum performance of $96.4 \%$ while the test performance has given 97\%. Strong efficiency and successful findings have been achieved. In the future, we will introduce other techniques for classification and prediction.

\section{REFERENCES}

[1] D. Q. Zeebaree, H. Haron, and A. M. Abdulazeez, "Gene Selection and Classification of Microarray Data Using Convolutional Neural Network," in ICOASE 2018 - International Conference on Advanced Science and Engineering, Nov. 2018, pp. 145-150, doi: 10.1109/ICOASE.2018.8548836.

[2] O. Ahmed and A. Brifcani, "Gene Expression Classification Based on Deep Learning Mobile operating systems View project Internet of things View project Gene Expression Classification Based on Deep Learning," doi: 10.1109/SICN47020.2019.9019357.

[3] D. Zebari, D. Zeebaree, J. N. Saeed, N. A. Zebari, D. A. Zebari, and D. Q. Zeebaree, "Significant features for steganography techniques using deoxyribonucleic acid: a review," Artic. Indones. J. Electr. Eng. Comput. Sci., vol. 21, no. 1, pp. 338347, 2021, doi: 10.11591/ijeecs.v21.i1.pp338-347.

[4] Z. Xie, Z. Guo, and C. Qian, "Palmprint gender classification by convolutional neural network," IET Comput. Vis., vol. 12, no. 4, pp. 476-483, 2018, doi: 10.1049/iet-cvi.2017.0475.

[5] J. $\mathrm{Hu}$, "An approach to EEG-based gender recognition using entropy measurement methods," Knowledge-Based Syst., vol. 140, pp. 134-141, 2018, doi: 10.1016/j.knosys.2017.10.032.

[6] M. R. Mahmood and A. M. Abdulazeez, "Different Model for Hand Gesture Recognition with a Novel Line Feature Extraction," in 2019 International Conference on Advanced Science and Engineering, ICOASE 2019, Apr. 2019, pp. 52-57, doi: 10.1109/ICOASE.2019.8723731.

[7] R. J.Hassan and A. M. Abdulazeez, "Deep Learning Convolutional Neural Network for Face Recognition: A Review," Int. J. Sci. Bus., vol. 5, no. 2, pp. 114127, 2021, Accessed: May 06, 2021. [Online]. Available:

https://ideas.repec.org/a/aif/journl/v5y2021i2p114127.html.

[8] B. Mohammed, S. Hasan, and A. Mohsin Abdulazeez, "Journal of Soft Computing and Data Mining A Review of Principal Component Analysis Algorithm for Dimensionality Reduction," J. SOFT Comput. DATA Min., vol. 2, no. 1, pp. 20-30, Apr. 2021, doi: 10.30880/jscdm.2021.02.01.003.

[9] N. Anne et al., "Feasibility and acceptability of an iris biometric system for unique patient identification in routine HIV services in Kenya," Int. J. Med. Inform., vol. 133, p. 104006, 2020, doi: 10.1016/j.ijmedinf.2019.104006.

[10] S. M. Saleem Abdullah and A. Mohsin Abdulazeez, "Journal of Soft Computing and Data Mining Facial Expression Recognition Based on Deep Learning Convolution Neural Network: A Review," J. SOFT Comput. DATA Min., vol. 2, no. 1, pp. 53-65, Apr. 2021, doi: 10.30880/jscdm.2021.02.01.006.

[11] D. M. Sulaiman, A. M. Abdulazeez, H. Haron, and S. S. Sadiq, "Unsupervised Learning Approach-Based New Optimization K-Means Clustering for Finger Vein Image Localization," in 2019 International Conference on Advanced Science and Engineering, ICOASE 2019, Apr. 2019, pp. 82-87, doi: 10.1109/ICOASE.2019.8723749.

[12] M. R. Christel-loïc TISSE, Lionel MARTIN, Lionel TORRES, "Person identification technique using human iris recognition," Vis. Interface, no. March, pp. 294-299, 2002.

[13] D. Zeebaree et al., "Multi-Level Fusion in Ultrasound for Cancer Detection Based on Uniform LBP Features Different Model for Hand Gesture Recognition with a Novel Line Feature Extraction View project Image Processing and Security View project Multi-Level Fusion in Ultrasound ," $C M C$, vol. 66, no. 3, 2021, doi: 10.32604/cmc.2021.013314.

[14] A. Mohsin Abdulazeez, D. Zeebaree, D. M. Abdulqader, and D. Q. Zeebaree, "Machine Learning Supervised Algorithms of Gene Selection: A Review Machine Learning View project How To Choose A Performance Metric View project Machine Learning Supervised Algorithms of Gene Selection: A Review," 2020. Accessed: May 06, 2021. [Online]. Available:

https://www.researchgate.net/publication/34111946 9.

[15] F. Q. Kareem and A. Mohsin Abdulazeez, "Ultrasound Medical Images Classification Based on Deep Learning Algorithms: A Review," vol. 3, no. 1, pp. 29-42, 2021, doi: 10.5281/zenodo.4621289.

[16] J. Najeeb Saeed and A. Mohsin Abdulazeez, "Journal of Soft Computing and Data Mining Facial Beauty Prediction and Analysis Based on Deep Convolutional Neural Network: A Review," J. SOFT Comput. DATA Min., vol. 2, no. 1, pp. 1-12, Apr. 2021, doi: 10.30880/jscdm.2021.02.01.001.

[17] D. Q. Zeebaree, H. Haron, A. M. Abdulazeez, and D. A. Zebari, "Trainable Model Based on New Uniform LBP Feature to Identify the Risk of the Breast Cancer," in 2019 International Conference on Advanced Science and Engineering, ICOASE 2019, Apr. 2019, pp. 106-111, doi: 10.1109/ICOASE.2019.8723827.

[18] S. Agatonovic-Kustrin and R. Beresford, "Basic concepts of artificial neural network (ANN) modeling and its application in pharmaceutical research," Journal of Pharmaceutical and Biomedical Analysis, vol. 22, no. 5. Elsevier, pp. 717-727, Jun. 01, 2000, doi: 10.1016/S07317085(99)00272-1.

[19] M. A. Alamir, “An artificial neural network model 
for predicting the performance of thermoacoustic refrigerators," Int. J. Heat Mass Transf., vol. 164, p. 120551, Jan. 2021, doi: 10.1016/j.ijheatmasstransfer.2020.120551.

[20] I. Mahmood Ibrahim and A. Mohsin Abdulazeez, "The Role of Machine Learning Algorithms for Diagnosing Diseases," jastt.org, vol. 02, no. 01, pp. 10-19, 2021, doi: 10.38094/jastt20179.

[21] H. Yahia, A. A.-I. J. of S. and, and undefined 2021, "Medical Text Classification Based on Convolutional Neural Network: A Review," ideas.repec.org, Accessed: May 06, 2021. [Online]. Available:

https://ideas.repec.org/a/aif/journl/v5y2021i3p2741.html.

[22] A. Jahwar, A. A.-P. J. of Archaeology, and undefined 2020, "PJAEE, 17 (7) (2021) MetaHeuristic Algorithms for K-means Clustering: A Review META-HEURISTIC ALGORITHMS FOR K-MEANS CLUSTERING: A REVIEW." Accessed: May 06, 2021. [Online]. Available: https://archives.palarch.nl/index.php/jae/article/view 14630.

[23] L. Xuan and Z. Hong, "An improved canny edge detection algorithm," in Proceedings of the IEEE International Conference on Software Engineering and Service Sciences, ICSESS, Apr. 2018, vol. 2017Novem, pp. 275-278, doi: 10.1109/ICSESS.2017.8342913.

[24] A. Kuehlkamp, "Predicting Gender From Iris Texture May Be Harder Than It Seems," arXiv, no. 1, pp. 19, 2018.

[25] K. Ito, H. Kawai, T. Okano, and T. Aoki, "Age and Gender Prediction from Face Images Using Convolutional Neural Network," 2018 Asia-Pacific Signal Inf. Process. Assoc. Annu. Summit Conf. APSIPA ASC 2018 - Proc., no. November, pp. 7-11, 2019, doi: 10.23919/APSIPA.2018.8659655.

[26] Sreya K C, “Gender Prediction from Iris Recognition using Artificial Neural Network (ANN)," Int. J. Eng. Res., vol. V9, no. 07, pp. 1214-1218, 2020, doi: 10.17577/ijertv9is070394.

[27] B. Fang et al., "Classification of genetically identical left and right irises using a convolutional neural network," Electron., vol. 8, no. 10, pp. 2-11, 2019, doi: 10.3390/electronics8101109.

[28] S. Arora and M. P. S. Bhatia, "A Robust Approach for Gender Recognition Using Deep Learning," 2018 9th Int. Conf. Comput. Commun. Netw. Technol. ICCCNT 2018, pp. 1-6, 2018, doi: 10.1109/ICCCNT.2018.8494194.

[29] A. Rattani, N. Reddy, and R. Derakhshani, "Convolutional neural networks for gender prediction from smartphone-based ocular images," IET Biometrics, vol. 7, no. 5, pp. 423-430, 2018, doi: 10.1049/iet-bmt.2017.0171.

[30] A. Grazia Mignani, L. Ciaccheri, A. A. Mencaglia, R. Di Sanzo, S. Carabetta, and M. Russo, "Dispersive raman spectroscopy for the nondestructive and rapid assessment of the quality of southern Italian honey types," J. Light. Technol., vol. 34, no. 19, pp. 44794485, 2016, doi: 10.1109/JLT.2016.2539550.
[31] M. Fang, N. Damer, F. Kirchbuchner, and A. Kuijper, "Demographic bias in presentation attack detection of iris recognition systems," in European Signal Processing Conference, Jan. 2021, vol. 2021-Janua, pp. 835-839, doi: 10.23919/Eusipco47968.2020.9287321.

[32] B. Muthazhagan and S. Sundaramoorthy, "Ameliorated Face and Iris Recognition Using Deep Convolutional Networks," in Studies in Computational Intelligence, vol. 919, Springer Science and Business Media Deutschland $\mathrm{GmbH}$, 2021, pp. 277-296.

[33] K. Bowyer, C. Kranenburg, and S. Dougherty, "Edge detector evaluation using empirical ROC curves," Comput. Vis. Image Underst., vol. 84, no. 1, pp. 77103, 2001, doi: 10.1006/cviu.2001.0931.

[34] X. Qin, “A modified Canny edge detector based on weighted least squares," Comput. Stat., vol. 36, no. 1, pp. 641-659, Mar. 2021, doi: 10.1007/s00180-02001017-8.

[35] J. Chouhan and A. Kulshrestha, "Analysis of Iris Detection Techniques," vol. 1, no. 5, pp. 1237-1241, 2019.

[36] D. Abas Hasan and A. Mohsin Abdulazeez, "A Modified Convolutional Neural Networks Model for Medical Image Segmentation Gait recognition with wavelet transform View project Different Model for Hand Gesture Recognition with a Novel Line Feature Extraction View project A Modified Convolutional Neur," Accessed: May 01, 2021. [Online]. Available: https://www.researchgate.net/publication/34310632 6.

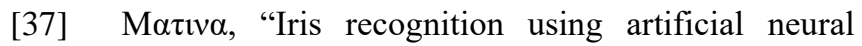
Networks and backpropagation," A $\gamma \alpha \eta$, vol. 8, no. 5, p. 55, 2019, [Online]. Available: http://docs.neu.edu.tr/library/6728644844.pdf.

[38] Y. Chen, C. Wu, and Y. Wang, "Whether normalized or not? Towards more robust iris recognition using dynamic programming," Image Vis. Comput., vol. 107, p. 104112, Mar. 2021, doi: 10.1016/j.imavis.2021.104112.

[39] M. Lavanya and V. Kavitha, "A hybrid classical techniques and optimal decision model for iris recognition under variable image quality conditions," J. Ambient Intell. Humaniz. Comput., pp. 1-19, Jan. 2021, doi: 10.1007/s12652-020-02691-8.

[40] A. Issa and A. Abdulazeez, "Intrusion Detection System Based on Neural Networks Using Bipolar Input with Bipolar Sigmoid Activation Function," AL-Rafidain J. Comput. Sci. Math., vol. 8, no. 2, pp. 79-86, Dec. 2011, doi: 10.33899/csmj.2011.163644.

[41] J. Gonçalves Neto, L. Vidal Ozorio, T. C. Campos de Abreu, B. Ferreira dos Santos, and F. Pradelle, "Modeling of biogas production from food, fruits and vegetables wastes using artificial neural network (ANN)," Fuel, vol. 285, p. 119081, Feb. 2021, doi: 10.1016/j.fuel.2020.119081.

[42] S. M. Saleem Abdullah and A. Mohsin Abdulazeez, "Journal of Soft Computing and Data Mining Facial Expression Recognition Based on Deep Learning Convolution Neural Network: A Review," J. SOFT Comput. DATA Min., vol. 2, no. 1, pp. 53-65, Apr. 
2021, doi: 10.30880/jscdm.2021.02.01.006.

[43] J. E. Tapia, C. A. Perez, and K. W. Bowyer, "Gender Classification From the Same Iris Code Used for Recognition," IEEE Trans. Inf. Forensics Secur., vol. 11, no. 8, pp. 1760-1770, 2016, doi: 10.1109/TIFS.2016.2550418.

[44] G. Mabuza-Hocquet, C. H. Ngejane, and S. Lefophane, "Predicting and Classifying Gender from the Human Iris: A Survey on Recent Advances," 2018 Int. Conf. Adv. Big Data, Comput. Data Commun. Syst. icABCD 2018, pp. 1-5, 2018, doi: 10.1109/ICABCD.2018.8465471.

[45] M. J. Barwary and A. M. Abdulazeez, "Impact of Deep Learning on Transfer Learning: A Review," Int. J. Sci. Bus., vol. 5, no. 3, pp. 204-216, 2021, Accessed: May 01, 2021. [Online]. Available: https://ideas.repec.org/a/aif/journl/v5y2021i3p204216.html.

[46] Y. Liu, X. Z. Pan, R. J. Shi, Y. L. Li, C. K. Wang, and Z. T. Li, "Predicting Soil Salt Content over Partially Vegetated Surfaces Using Non-Negative Matrix Factorization," IEEE J. Sel. Top. Appl. Earth Obs. Remote Sens., vol. 8, no. 11, pp. 5305-5316, 2015, doi: 10.1109/JSTARS.2015.2478490.

[47] A. Kumar and A. Passi, "Comparison and Combination of Iris Matchers for Reliable Personal Authentication," 2010.

[48] "Deep Convolution Neural Network for Facial Expression Recognition | PalArch's Journal of Archaeology of Egypt / Egyptology." https://archives.palarch.nl/index.php/jae/article/view /6874 (accessed May 01, 2021).

[49] Y. Su, "Target detection algorithm and data model optimization based on improved Gaussian mixture model," Microprocess. Microsyst., vol. 81, p. 103797, Mar. 2021, doi: 10.1016/j.micpro.2020.103797.

[50] H. Kang and I. Stamoulis, "Gaussian Image Binarization," Int. J. Image Graph., Mar. 2021, doi: 10.1142/S0219467821500479.

[51] N. Fatema and H. Malik, "Data-driven occupancy detection hybrid model using particle swarm optimization based artificial neural network," in Studies in Computational Intelligence, vol. 916, Springer Science and Business Media Deutschland GmbH, 2021, pp. 283-297.

[52] M. R. Mahmood, "A NEW HAND GESTURE RECOGNITION SYSTEM USING ARTIFICIAL NEURAL NETWORK Facial Expression Recognition by using Relief-F Feature Selection classified by J48 KNN RBF SVM MLP RF View project Cloud Computing Resources Impacts on Heavy-Load Parallel Processing A,” 2017. Accessed: May 01, 2021. [Online]. Available: https://www.researchgate.net/publication/32085348 5.

[53] M. I. Jahirul et al., "Investigation of correlation between chemical composition and properties of biodiesel using principal component analysis (PCA) and artificial neural network (ANN)," Renew. Energy, vol. 168, pp. 632-646, May 2021, doi: 10.1016/j.renene.2020.12.078.
[54] J. Tapia, C. Arellano, and I. Viedma, "Sexclassification from cellphones periocular iris images," in Advances in Computer Vision and Pattern Recognition, Springer London, 2019, pp. 227-242.

[55] C. O. Lawal, A. A. Akinrinmade, and J. A. Badejo, "Face-based Gender recognition Analysis for Nigerians Using CNN," in Journal of Physics: Conference Series, Dec. 2019, vol. 1378, no. 3, p. 032014, doi: 10.1088/1742-6596/1378/3/032014.

[56] M. Sandhya et al., "Deep Neural Networks with Multi-class SVM for Recognition of Cross-Spectral Iris Images," in Communications in Computer and Information Science, 2021, vol. 1366, pp. 29-41, doi: 10.1007/978-981-16-0419-5_3.

[57] S. Ullah, M. J. Khan, K. Khurshid, and M. Ul Hasan, "Comparative Analysis of SVM, ANN and CNN for Classifying Vegetation Specie using Hyperspectral Thermal Infrared Data COMPARATIVE ANALYSIS OF SVM, ANN AND CNN FOR CLASSIFYING VEGETATION SPECIES USING HYPERSPECTRAL THERMAL INFRARED DATA," 2019, doi: 10.5194/isprs-archives-XLII-2W13-1861-2019.

[58] O. Deperlioglu, "Classification of Phonocardiograms with Convolutional Neural Networks," May 2018. Accessed: May 01, 2021. [Online]. Available: https://www.brain.edusoft.ro/index.php/brain/article /view/804.

[59] Ş. Öztürk and U. Özkaya, "Gastrointestinal tract classification using improved LSTM based CNN," Multimed. Tools Appl., vol. 79, no. 39-40, pp. 28825-28840, Oct. 2020, doi: 10.1007/s11042-02009468-3.

[60] "Evaluating Data Mining Classification Methods Performance in Internet of Things Applications | Journal of Soft Computing and Data Mining." https://publisher.uthm.edu.my/ojs/index.php/jscdm/a rticle/view/7127 (accessed May 01, 2021).

[61] Y. Sun, M. Zhang, Z. Sun, and T. Tan, "Demographic Analysis from Biometric Data: Achievements, Challenges, and New Frontiers," IEEE Trans. Pattern Anal. Mach. Intell., vol. 40, no. 2, pp. 332351, 2018, doi: 10.1109/TPAMI.2017.2669035.

[62] S. Lagree and K. W. Bowyer, "Predicting ethnicity and gender from iris texture," 2011 IEEE Int. Conf. Technol. Homel. Secur. HST 2011, pp. 440-445, 2011, doi: 10.1109/THS.2011.6107909.

[63] "GAIT-BASED HUMAN GENDER CLASSIFICATION USING 5/3 LIFTING BASED WAVELET FILTERS AND PRINCIPAL COMPONENT ANALYSIS." https://acikerisim.siirt.edu.tr/xmlui/handle/20.500.1 2604/2092 (accessed May 01, 2021). 